\title{
Analytical Method Validation
}

\author{
Pedro Lopez Garcia ${ }^{1}$, Ernesto Buffoni ${ }^{1}$, \\ Fabio Pereira Gomes ${ }^{1}$ and Jose Luis Vilchez Quero ${ }^{2}$ \\ ${ }^{1}$ Instituto de Aperfeiçoamento Farmacêutico (IAF) \\ ${ }^{2}$ Department of Analytical Chemistry, Faculty of Sciences, University of Granada \\ ${ }^{1}$ Brazil \\ 2Spain
}

\section{Introduction}

In pharmaceutical industries, the validation of analytical methods is used to demonstrate that the method is fitted for its purpose; it must follow a plan which includes scope, performance characteristics, and acceptance limits. Analytical methods need to be validated or revalidated prior to their introduction into routine analyses (release of batch). The overarching philosophy in current good manufacturing practices (cGMPs) of the twenty first century and robust modern quality systems is the quality that it has to be built into the product, and testing alone cannot be relied to ensure the quality of the product. From the analytical perspective, it will mean that analytical methods used to test products should have quality attributes built into them. In order to apply quality attributes into the analytical method, fundamental quality attributes have to be applied by the bench - level scientist. This is a paradigm shift that requires the bench - level scientist to have a scientific and technical understanding, product knowledge, process knowledge, and/or risk assessment ability to appropriately execute the quality functions of analytical method validation. In addition, it requires the following procedures: (a) an appropriate training of the bench - level scientist to understand the principles involved with method validation, validate an analytical method, and understand the principles involved with the method validation, (b) proper documentation and understanding and interpreting data, and (c) cross - an understanding functional of the effect of their activities on the product and to customers (the patient). Management has a responsibility of verifying that gained skills from the training are implemented in routine analyses performance.

This chapter gives a review and strategy for the validation of analytical methods in-house, recommendation in documentation and completion of method validation in the pharmaceutical environmental.

\section{Regulatory agencies}

In 1990, Europe, United States of America, and Japan harmonized the submission requirements for new pharmaceuticals, a forum for constructive dialogues between regulatory authorities and industry was initiated and called, "International Conference on the Harmonisation" (ICH) (ICH, 2005). One of the first topics into the quality section was 
analytical validation and the ICH was very helpful in harmonizing terms and definitions as well as determining the basic requirements.

Regulatory authorities in the United States of America (Food and Drug Administration (FDA) and the United States Pharmacopeia (USP)) and Brazil (Agência Nacional de Vigilância Sanitária (ANVISA)) include guidelines for analytical procedures and methods validation. In the United States of America environment, two guidelines by the FDA were proposed, one of those for applicants (Food and Drugs Administration, 1987) and another one for inspectors and reviewers (Food and Drugs Administration, 1994). The first one is also intended to ensure that the analytical procedures have to be applied in an FDA laboratory with detailed description of the procedure, for instance, reference materials, as well as a discussion of the potential impurities, and others. The second guideline focuses on reversed-phase chromatography and provides the whole details regarding critical methodological issues as well as indications of acceptability results. The USP has published a specific guideline in the chapter 1225. It focuses on the validation of compendia procedures by giving definitions and approaches to validate each analytical parameter, in addition, it also provides a table which separates the methods into four categories based on their use (United States Pharmacopeia, 2011). In Brazil, ANVISA has also proposed an industry guidance for analytical methods validation. The RE 899 of 2003 (Brasil, 2003) is an approach to validation and it is varied and opened to interpretation. Also it provides a table, the same as chapter 1225 of the USP which separates methods into four categories based on their use. For example, Category I covers quantitation of active ingredients in dosage forms and indicates that accuracy, precision, specificity, linearity, and range are required for method validation while limits of detection and quantitation are not necessary.

The USP 1225 chapter covers only the most common categories of tests which validation data should be required. Those categories can be observed on the following lines:

Category I: Analytical procedures for quantitation of major components in bulk drug substances or active ingredients (including preservatives) in finished pharmaceutical products.

Category II: Analytical procedures for determination of impurities in bulk drug substances or degradation compounds in finished pharmaceutical products. These procedures include quantitative assays and limit tests.

Category III: Analytical procedures for determination of performance characteristics (e.g., dissolution, drug release, etc.).

Category IV: Identification tests.

In Table 1, the required validation characteristics for various types of analytical procedures according to USP can be observed.

The required validation characteristics for each type of analytical procedures according to ICH are illustrated in Table 2. The discussion of the validation of analytical procedures is directed to the four most common types of analytical procedures:

- Identification tests.

- Quantitative tests for impurities' content.

- Limit tests for the control of impurities.

- Quantitative tests of the active moiety in samples of drug substance or drug product or other selected component(s) in the drug product.

Even though there are many other analytical procedures, such as dissolution testing for drug products or particle size determination for drug substance, these are no addressed in the initial text for validation of analytical procedures. The validation of these additional 
analytical procedures is equally important to those listed herein, and it may be addressed in subsequent documents.

\begin{tabular}{cccccc}
\hline $\begin{array}{c}\text { Analytical } \\
\text { performance } \\
\text { characteristics }\end{array}$ & Category I & \multicolumn{2}{c}{ Category II } & Category III & Category IV \\
\hline Accuracy & Yes & Yes & $*$ & $*$ & No \\
Precision & Yes & Yes & No & Yes & No \\
Specificity & Yes & Yes & Yes & $*$ & Yes \\
Detection Limit & No & No & Yes & $*$ & No \\
Quantitation Limit & No & Yes & No & $*$ & No \\
Linearity & Yes & Yes & No & $*$ & No \\
Range & Yes & Yes & $*$ & $*$ & No \\
\hline
\end{tabular}

*It may be required, depending on the nature of the specific test.

Table 1. Data elements required for validation according to USP

\begin{tabular}{lcccc}
\hline $\begin{array}{l}\text { Type of analytical } \\
\text { procedure }\end{array}$ & Identification & \multicolumn{2}{c}{ Testing for impurities } & Assay \\
Characteristics & - & Quantitative & Limit & \\
\hline Accuracy & - & + & - & + \\
Precision & - & + & & + \\
Repeatability & + & $+(1)$ & - & $+(1)$ \\
Interm. precision & - & + & + & + \\
Specificity (2) & - & $-(3)$ & + & - \\
Detection Limit & - & + & - & - \\
Quantitation Limit & - & + & - & + \\
Linearity & - & + & - & + \\
Range & & & + & - \\
\hline
\end{tabular}

(-) characteristic is not normally evaluated. $(+)$ characteristic is normally evaluated.

(1) in some cases where reproducibility (see glossary) has been performed, intermediate precision does not need to be done. (2) lack of specificity in one analytical procedure could be compensated by other supporting analytical procedure(s). (3) it may be needed in some cases.

Table 2. Data elements required for validation according to $\mathrm{ICH}$

A brief description of the types of considered tests in this document is provided below.

- Identification tests are intended to ensure that analyte is into of sample. This is normally achieved by comparing the property of the sample (e.g., spectrum, chromatographic behavior, chemical reactivity, etc) with a reference standard.

- Testing for impurities can be either a quantitative test or limit test for the impurity in a sample. Both tests are intended to accurately reflect the purity characteristics of the 
sample. More different validation characteristics are required for a quantitative than for a limit test.

- Assay procedures are intended to measure the presented analyte in a given sample. In the context of this document, the assay represents a quantitative measurement of the major component(s) in the drug substance. For the drug product, similar validation characteristics are also applied when assaying an active or other selected component(s). The same validation characteristics may also be applied to associated assays with other analytical procedures (e.g., dissolution).

In Brazil, ANVISA has the same form as ICH and USP, it covers the most common categories of tests for analytical method validation: category I, category II, category III, and category IV. The Table 3 shows performance characteristics of an analytical method validation according to resolution RE 899.

\begin{tabular}{lccccc}
\hline \multirow{2}{*}{ Parameter } & \multirow{2}{*}{ Category I } & \multicolumn{2}{c}{ Category II } & \multirow{2}{*}{ Category III } & \multirow{2}{*}{ Category IV } \\
& Quantitative & Limit tests & \multirow{2}{*}{ Yes } \\
Specificity & Yes & Yes & Yes & $*$ & No \\
Linearity & Yes & Yes & No & $*$ & No \\
Range & Yes & Yes & $*$ & $*$ & No \\
Recision & & & & & Yes \\
Interm. precision & Yes & Yes & No & $* *$ & No \\
Detection Limit & No & No & Yes & $*$ & No \\
Quantitation Limit & No & Yes & No & $*$ & No \\
Accuracy & Yes & Yes & $*$ & $*$ & No \\
Robustness & Yes & Yes & Yes & No & \\
\hline
\end{tabular}

* it may be required, depending on the nature of the specific test.

** If there is evidence of reproducibility is not required intermediate precision.

Table 3. Data elements required for validation according to RE 899 of ANVISA

\section{Strategy for analytical method validation}

The validation of a specific method must be demonstrated through laboratory experiments by routinely analyzing samples. The preparation and execution have to follow a validation protocol, preferably written in a step-by-step instruction format. Possible steps for a complete method validation are listed in Table 4 . This proposed procedure assumes that the instrument has been selected, and the method has also been developed. It meets criteria such as easy of use, ability to be automated and controlled by computer systems, costs per analysis, sample throughput, turnaround time, environmental, health, and safety requirements. Successful acceptance of the validation parameters and performance criteria by all involved parties requires a cooperative effort of several departments, including analytical development, quality control, regulatory affairs, and individuals requiring the analytical data. The operating procedure or Validation Master Plan (VMP) must clearly define the rules and responsibilities of each involved department in the validation of analytical methods. 
The validation experiments should be carried out by an expert analyst to avoid errors due to lack of experience. The analyst should also be very familiar with the technique and operation of the instrument. Before an instrument starting validating a method, its performance specifications must be verified by using generic chemical standards. Satisfactory results for a method can be only obtained with equipment that is performing well. A special attention must be paid to equipment characteristics which are critical for the method. For instance, if the detection limit is critical for a specific method, the specification of the instrument for baseline noise and some detectors must be verified.

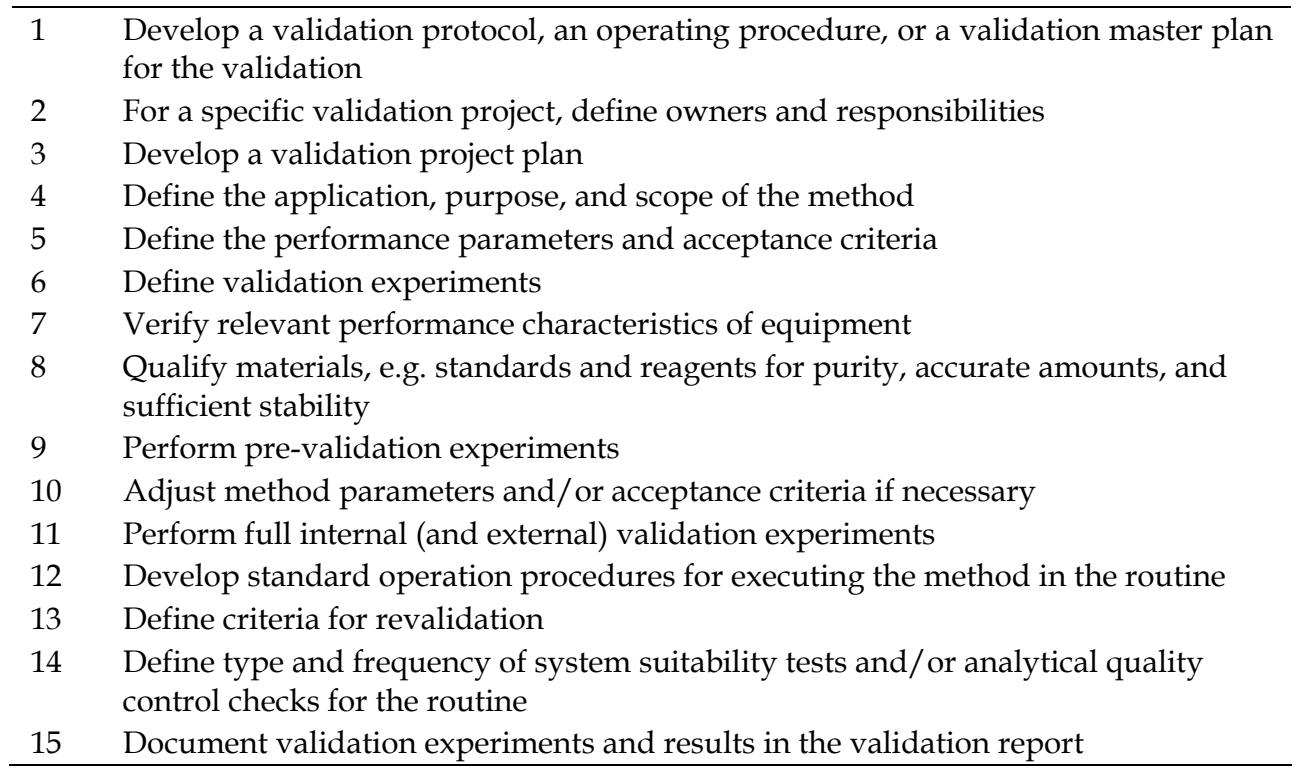

Table 4. Strategies and steps in analytical method validation

There are no official guidelines on the correct sequence of validation experiments, and the optimal sequence may depend on the method itself.

Based on the experience of, the following sequence has proven to be very helpful:

1. Specificity (optimizing separation and detection of standard mixtures if selectivity is insufficient).

2. Linearity, range, limit of detection, and limit of quantitation.

3. Precision (Repeatability, short-term precision, intermediate precision, and reprodutibility).

4. Accuracy at different concentrations.

5. Robustness.

The time-consuming experiments, such as accuracy and ruggedness, are included toward the end. Some of the parameters, as listed under (2) to (6), can be measured by combining experiments. For example, when the precision of peak areas is measured over the full concentration range, the data can be used to validate the linearity. During method validation, the parameters, acceptance limits, and frequency of ongoing system suitability tests or quality control checks should be defined. Criteria must be defined to indicate when the method and system are beyond statistical control. The aim is to optimize these 
experiments so that, with a minimum number of control analyses, the method and the complete analytical system will provide long-term results to meet the objectives defined in the scope of the method.

\section{Documentation}

\subsection{Validation protocol}

Depending upon of the culture of the company, a method validation protocol could be simple (listed below) or exhaustive (in addition to the listed items, each parameter to be validated is described in detail): How solutions are going to be made, the experimental design, how the calculations are going to be performed, any software to be utilized (e.g., Excel). If a full-length protocol is required within a particular company, then the writing of this protocol and approval of the protocol would need to be completed prior to the commencement of the validation work. Otherwise, there may be many deviations to the protocol which will be needed to be referenced in the final method validation report. Some companies also have templates for the validation reports, thereby allowing for facile population of the results. Once populated, the file is reviewed to determine if all validation parameters and acceptance criteria were met. If they were not met, a deviation is added and the proper justification must be given. If it is deemed that the justification is not appropriate, then an action plan for the specific figure of merit in question is determined (i.e., repeat analysis, change of the analytical procedure, and revalidation). Also, if the analytical method has not been approved at the time of writing the validation protocol yet, it is recommended to attach a final draft of the method to the protocol.

Before starting the experimental work, the protocols must be written by a qualified person and approved by a quality assurance department. Some of the items that are necessary to be specified in the validation protocol are listed below:

- The analytical method for a given product or drug substance.

- The test to be validated.

- The test parameters for each test, including type and number of solutions and number of tests.

- The acceptance criteria for each parameter based on an internal standard operational procedure (product or method-specific adaptations may be necessary and are acceptable, if justified).

- List of batches of drug substance and/or drug products.

- For a drug product the grade/quality of the excipients used in the formulation.

- List of reference materials to be used in the validation experiments.

- Information of the instruments and apparatus to be used.

- Responsibilities [author, chemists, analytical research project leader, quality assurance, and etc.].

\subsection{Validation report}

The validation report must contain reference to the analytical methods (specific code number used as identifier within the pharmaceutical organization) and the corresponding drug substance or product name. Note that for early-phase method validation reports the results may be filled in a predefined table and compared with the acceptance criteria. However, for late-phase validation, more explicit reports are generated by explaining every experiment, with detailed steps of sample and standard preparation. The list of reference materials (reference standards with the appropriate certificate of analysis) as well as the list 
of calibrated and qualified instruments used in the validation experiments should be documented in the report. For drug substances the list of the batches of drug substances, notebook number/reference number for any individual impurities, or solutions or used intermediates should also be listed. For drug products, the list of the batches of drug substances, drug product, and the grade/quality of excipients should be listed. The test parameters and acceptance criteria must be listed together with the results for each test, and the passed or failed results should be indicated. The validation report should also contain whether the method validation was successful and if any changes should be applied to the analytical method, and then the final analytical method must be resubmitted for quality assurance approval.

Once the method has been developed and validated, a validation report should be prepared with the following information:

- $\quad$ Objective and scope of the method (applicability, type).

- Summary of methodology.

- All chemicals, reagents, reference standards, quality control samples with purity, grade, their source, or detailed instructions on their preparation.

- Procedures for quality checks of standards and chemicals used on that.

- Safety precautions.

- Method parameters.

- Critical parameters from robustness testing.

- Listing of equipment and its functional and performance requirements, e.g., cell dimensions, baseline noise, and column temperature range. For complex equipment, a picture or schematic diagram may be useful.

- Detailed conditions on how the experiments were conducted, including sample preparation. The report must be detailed enough to ensure that it can be reproduced by a competent technician with comparable equipment.

- Statistical procedures and representative calculations.

- Procedures for quality control in routine analyses, e.g., system suitability tests.

- Representative plots, e.g., chromatograms, spectra, and calibration curves.

- Method acceptance limit performance data.

- The expected uncertainty of measurement results.

- Criteria for revalidation.

- The person(s) who developed and validated the method.

- References (if any).

- Summary and conclusions.

- Approval with names, titles, date, and signature of those responsible for the review and approval of the analytical test procedure.

\subsection{Revalidation}

Some method parameters have to be changed or adjusted during the life of the method if the method performance criteria fall outside their acceptance criteria. The question is whether such change requires revalidation. In order to clarify this question up front, operating ranges should be defined for each method, either based on experience with similar methods or else investigated during method development. These ranges should be verified during method validation in robustness studies and also be part of the method characteristics. Availability of such operating ranges makes it easier to decide when a method should be 
revalidated. A revalidation is necessary whenever a method is changed and the new parameter lies outside the operating range. If, for example, the operating range of the column temperature has been specified to be between $30^{\circ} \mathrm{C}$ and $40^{\circ} \mathrm{C}$, the method should be revalidated if, for whatever reason, the new operating parameter is $41^{\circ} \mathrm{C}$. Revalidation is also required if the scope of the method has been changed or extended, for instance, if the sample matrix changes or operating conditions change. Furthermore, revalidation is necessary whether the intention is to use instruments with different characteristics and these new characteristics have not been covered by the initial validation. For example, an HPLC method may have been developed and validated on a pump with a delay volume of $5 \mathrm{~mL}$, but the new pump has a delay volume of only $0.5 \mathrm{~mL}$. Part or full revalidation may also be considered if system suitability tests, or the results of quality control sample analysis, lie outside preset acceptance criteria where the source of the error cannot be traced back to the instruments or any other cause. Whenever there is a change that may require part or full revalidation, the change should follow a documented change control system. The change should be defined, authorized for implementation, and documented. Possible changes may include:

- New samples with new compounds or new matrices.

- New analysts with different skills.

- New instruments with different characteristics.

- New location with different environmental conditions.

- New chemicals and/or reference standards, and

- Modification of analytical parameters.

An evaluation should determine whether the change is within the scope of the method. If so, no revalidation is required. If the change lies outside the scope, the parameters for revalidation should be defined. After the validation experiments, the system suitability test parameters should be investigated and redefined, if necessary.

\section{Parameters for method validation (figures of merit)}

The type and degree of validation depends on the nature of the test. In particular, methods described in pharmacopeias may not have to be validated, but those should be verified. Different test methods require different validation parameters; as development of the project progresses and as analytical and product-specific information is acquired, the analytical methods evolve gradually updated. Each company has its own approach and set of acceptance criteria for different analytical assays, but these approaches must be within the confines of their line unit quality assurance department and be in accordance with any regulatory provisions. In this section, a description for each of the parameters to be validated (figures of merit) is described in details.

\subsection{Specificity}

An investigation of specificity should be conducted during the validation of identification test, the determination of impurities, and the assay. The procedures used to demonstrate specificity will depend on the intended objective of the analytical procedure. It is not always possible to demonstrate that an analytical procedure is specific for a particular analyte (complete discrimination). In this case, a combination of two or more analytical procedures is recommended to achieve the necessary level of discrimination (e.g., optically active substances, in addition to an achiral HPLC method, a chiral HPLC method may be added). 
Identity is a general requirement for dosage forms. When determining specificity for identity, the assay and related substances or the content uniformity methods can be used. Assay and content uniformity methods are quantitated by external reference standard. This identity test confirms that the correct active ingredient (s) is presented in correct ratio if multiple variants are available. The method could also be used for post-packaging analysis. The general requirements are that the sample and standard chromatograms should correspond in retention time and normalized peak area within $\pm 10 \%$. The easiest way to perform specificity for any HPLC method is to perform this test in conjunction with a forced decomposition study. The utilization of mass spectrometry (MS) detector (in series) after a Photo Diode Array (PDA) detector to obtain more information is encouraged (in terms of mass-to-charge ratio of parent ions, initial fragmentation pattern, and peak purity). Specificity is confirmed when an API peak is "pure" (confirmed by PDA and/or MS) and there is no interference from placebo solution (placebo dissolved in sample preparation solvent) at the retention time of an API peak.

\subsubsection{Forced degradation studies (solid state and solution)}

Forced degradation studies are usually performed during the salt selection process for the drug substance. In drug product development, the forced degradation studies of drug substance in the presence of excipients are firstly performed during the pre-formulation stage to assist in the selection of the most formidable compounds and excipients. This may lead to the development of more suitable formulations, packaging, and change in storage and manufacturing conditions as the optimal formulation is defined to be used in clinical studies. Forced degradation testing is often repeated when the final drug substance route and market formulation is defined or the compound enters phase 3 clinical trials. A good overview of forced degradation testing according to the regulatory guidance documents, with emphasis on what should be considered for late clinical phases and for registration application dossiers (i.e., marketing authorization applications or new drug applications), is provided by the Impurity Profiling Group (Klick et al., 2005). Forced degradation studies (sometimes referred to as stress testing) are also performed in order to demonstrate specificity during the development and validation of stability-indicating methods. These studies are usually performed at conditions exceeding that of accelerated storage conditions. Forced degradation studies may provide information to degradation pathways and degradation products that could form during storage of the drug substance or the drug product. The main goal of forced degradation studies is to effectively produce samples containing representative and realistic degradation products. These degradation products should be assessed whether they are (a) related to the drug substance or the excipients or (b) due to drug substance-excipient interactions under certain forced degradation conditions.

A delicate balance of efficiency and severity/duration of stress conditions is needed. Overstressing can destroy relevant compounds or generate irrelevant compounds. Under stressing may fail to generate important degradation products. The extent of targeted degradation should be approximately anywhere from $5 \%$ to $10 \%$. The other goal is that the potential degradation products that are generated should be resolved from the active component during development of a stability-indicating HPLC method. The assessment of peak purity using diode array and LC-MS detection are usually employed. These degradation products that are generated during the forced degradation studies can be identified, and the determination of degradation pathways and mechanisms for the drug substance and drug product can be elucidated. Forced degradation studies are carried out either in the solution 
state and/or in the solid state. Usually the forced degradation testing is carried out on one batch of drug substance and/or one formulation blend (capsules and tablets). This forced degradation testing should not be part of a formal stability program.

\begin{tabular}{lll}
\hline Stress Test & Conditions & Duration \\
\hline $\begin{array}{l}\text { Thermal } \\
\text { (closed container) }\end{array}$ & $\begin{array}{l}50^{\circ} \mathrm{C} \text { and } 80^{\circ} \mathrm{C} \\
(\mathrm{ambient} \mathrm{RH})\end{array}$ & $1 \mathrm{wk}$ and $2 \mathrm{wks}$ \\
$\begin{array}{l}\text { Thermal/oxidative } \\
\text { (open container) }\end{array}$ & $\begin{array}{l}50^{\circ} \mathrm{C} \text { and } 80^{\circ} \mathrm{C} \\
(\text { ambient } \mathrm{RH})\end{array}$ & $1 \mathrm{wk}$ and $2 \mathrm{wks}$ \\
$\begin{array}{l}\text { Thermal/humidity } \\
\text { (open container) }\end{array}$ & $40^{\circ} \mathrm{C} / 75 \% \mathrm{RH}$ & $1 \mathrm{wk}$ and $2 \mathrm{wks}$ \\
$\begin{array}{l}\text { Light } \\
\text { (closed container) }\end{array}$ & Ambient & $\begin{array}{l}\text { Maximum } 1.2 \text { million lux hours } \\
\text { and } 200 \text { watt hours/square meter }\end{array}$ \\
$\begin{array}{l}\text { Light/oxidative } \\
\text { (open container) }\end{array}$ & Ambient & $\begin{array}{l}\text { Maximum } 1.2 \text { million lux hours } \\
\text { and } 200 \text { watt hours / square meter }\end{array}$ \\
\hline
\end{tabular}

Table 5. Solid-state forced degradation studies

\begin{tabular}{|c|c|c|}
\hline Test factor & Conditions & Duration \\
\hline $\mathrm{pH}$ & $\begin{array}{l}10 \mathrm{mg} \text { in } 2 \mathrm{~mL} \text { water } \\
10 \mathrm{mg} \text { in } 2 \mathrm{~mL} \text { of } 0.1 \mathrm{M} \mathrm{HCl} \\
10 \mathrm{mg} \text { in } 2 \mathrm{~mL} \text { of } 0.1 \mathrm{M} \mathrm{NaOH} \\
\text { All in amber volumetric flasks and } \\
\text { at room temperature }\end{array}$ & 1 day and 3 days \\
\hline $\begin{array}{l}\text { Oxidation } \\
\left(\mathrm{H}_{2} \mathrm{O}_{2}\right)\end{array}$ & $\begin{array}{l}10 \mathrm{mg} / 2 \mathrm{~mL} 3 \% \mathrm{H} 2 \mathrm{O} 2 \\
\text { At } 5^{\circ} \mathrm{C} \text { and room temperature in } \\
\text { amber volumetric flasks. If DS is } \\
\text { not soluble, then pH modification } \\
\text { may be necessary }\end{array}$ & 1,2 , and 3 days \\
\hline $\begin{array}{l}\text { Oxidation } \\
\text { (metal ion catalyzed) }\end{array}$ & $\begin{array}{l}10 \mathrm{mg} / 2 \mathrm{~mL} \text { water containing } \\
\text { 100ppm } \mathrm{Fe} 3+, \mathrm{Ni} 2+, \mathrm{Cu} 2+ \\
\text { saturated with bubbled oxygen in } \\
\text { amber volumetric flasks }\end{array}$ & 1,2 , and 3 days \\
\hline $\begin{array}{l}\text { Oxidation } \\
\text { (saturated with oxygen) }\end{array}$ & $\begin{array}{l}10 \mathrm{mg} / 2 \mathrm{~mL} \text { saturated with bubbled } \\
\text { oxygen in amber volumetric flasks }\end{array}$ & 1,2 , and 3 days \\
\hline Light & $50 \mathrm{mg} / 10 \mathrm{~mL}$ wáter ambient & $\begin{array}{l}\text { Maximum } 1.2 \text { million } \\
\text { lux hours and } 200 \text { watt } \\
\text { hours/square meter, } 6 \mathrm{hr} \text {, } \\
1 \text { day and } 2 \text { days }\end{array}$ \\
\hline Heat & $10 \mathrm{mg}$ in $2 \mathrm{~mL}$ water at $50^{\circ} \mathrm{C}$ & 6 hours, 1 day and 2 days \\
\hline
\end{tabular}

Table 6. Solution forced degradation studies 
The temperature/humidity used conditions may be more severe than the typical accelerated stability testing conditions in order to generate potential degradation products in a reasonable time. The typical forced degradation conditions include thermolytic, hydrolytic, oxidative, photolytic (in excess of ICH conditions), high $\mathrm{pH}$ (alkaline conditions), and low $\mathrm{pH}$ (acidic conditions). Outlined in Table 5 and Table 6 are some solid-state and solution forced degradation studies, respectively, that could be conducted.

\subsection{Linearity}

The linearity of an analytical procedure is its ability (within a given range) to obtain test results which are directly proportional to the concentration (amount) of analyte in the sample. It may be directly demonstrated on the analyte, or on spiked samples using at least five concentrations over the whole working range. Moreover a visual evaluation of the analyte signal as a function of the concentration, appropriate statistical calculations are recommended, such as a linear regression. The parameters slope and intercept, residual sum of squares and the coefficient of correlation should be reported. A graphical presentation of the data and residuals is recommended.

The terminology for this validation characteristic is somewhat misleading because linearity in the inner sense, i.e., a linear relationship between analyte concentration and test results is certainly preferable, but not essential. A better term would have been analytical response. Some analytical procedures have intrinsic non-linear response functions, such as quantitative TLC, fluorescence detection, etc., but they can of course be validated. The primary objective is to validate or verify the calibration model. Consequently, the requirements and the relevant parameters depend on the intended mode of calibration. The response function of a given analytical procedure is an intrinsic property of the respective analyte. That means, with respect to validation, that the answer is of a qualitative kind: Can the intended calibration be applied, yes or no? Therefore, solutions of the analyte itself are sufficient and there is no need to repeat linearity. Potential influences by the matrix, i.e., the linearity of the analytical procedure would be better addressed in accuracy. Often, the fundamental response function is known for a given type of analytical procedure, such as a linear function for LC with UV detection, according to the Lambert-Beer law. In some cases, validation of linearity can be regarded more as a verification of the assumed response function, i.e., the absence of (unacceptable) deviations. Primarily, this should be performed by means of graphical evaluation of the deviations of the experimental data from the assumed response model (residual analysis), known as residual plots. The evaluation of numerical parameters is only sensible after verification of the response function, i.e., if only random errors exist.

Frequently, the linearity is evaluated graphically, in addition to or as an alternative to mathematical evaluation. The evaluation is made by visually inspecting a plot of signal height or peak area as a function of analyte concentration. Because deviations from linearity are sometimes difficult to detect, two additional graphical procedures can be used. The first is to plot the deviations from the regression line versus the concentration or versus the logarithm of the concentration if the concentration range covers several decades. For linear ranges, the deviations should be equally distributed between positive and negative values. Another approach is to divide signal data by their respective concentrations, yielding the relative responses. A graph is plotted with the relative responses on the y-axis and the corresponding concentrations on the x-axis, on a log scale. The obtained line should be horizontal over the full linear range. At higher concentrations, there will typically be a 
negative deviation from linearity. Parallel horizontal lines are drawn on the graph corresponding, for example, 95 percent and 105 percent of the horizontal line. The method is linear up to the point where the plotted relative response line intersects the 95 percent line. Figure 1 shows a comparison of the two graphical evaluations on a sample of caffeine using HPLC.

A plot of the data should be included in the report. In addition, an analysis of the deviation of the actual data points from the regression line may also be helpful for evaluating linearity. In order to establish linearity, a minimum of five concentrations is recommended. Other approaches should be justified. Plotting the sensitivity (response/amount) gives clear indication of the linear range. Plotting the amount on a logarithmic scale has a significant advantage for wide linear ranges.
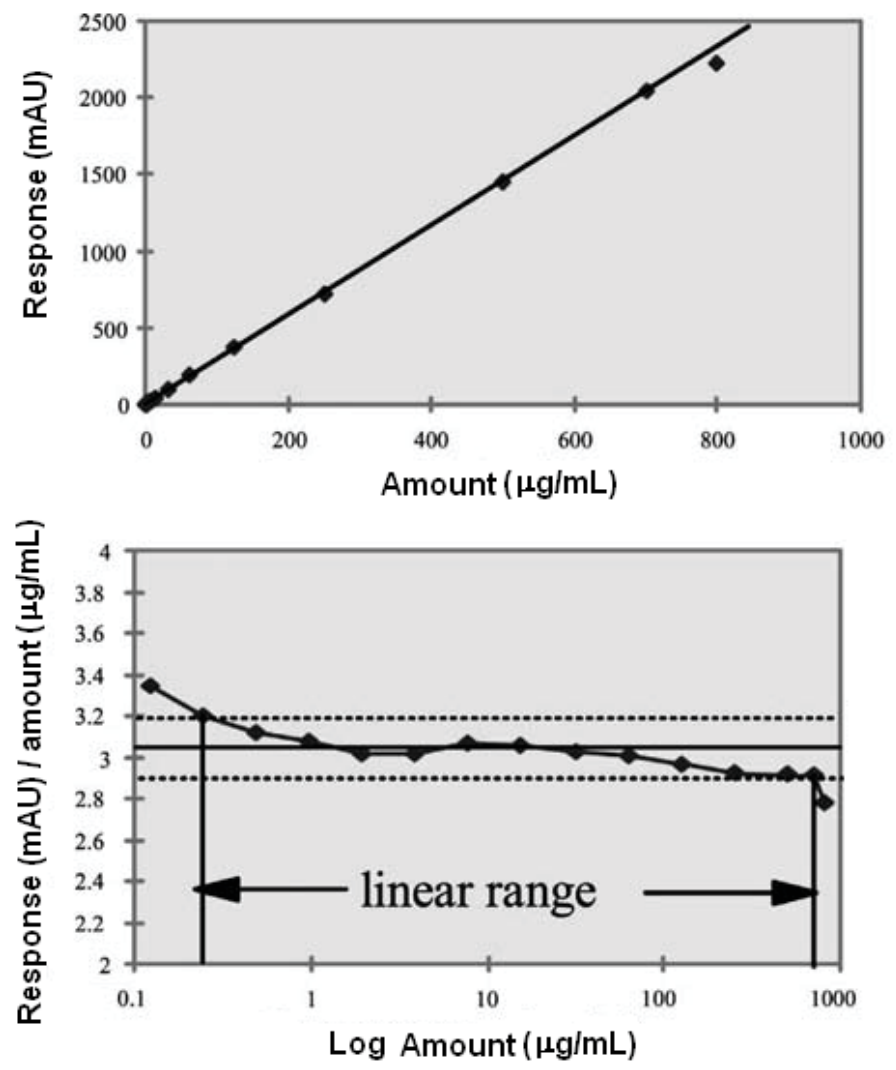

Fig. 1. Linearity plot of an analyte sample

\subsubsection{Range}

The range of an analytical method is the interval between the upper and lower levels (including these levels) that have been demonstrated to be determined with precision, accuracy, and linearity using the method as written. The range is normally expressed in the same units as the test results. For assay test, it requires a minimum of specified range to be 
80 to 120 percent of the test concentration, and for the determination of an impurity, the range to extend from the limit of quantitation, or from 50 percent of the specification of each impurity, whichever is greater to 120 percent of the specification.

\subsubsection{Limit of detection}

The limit of detection is the point at which a measured value is larger than the uncertainty associated with it. It is the lowest concentration of analyte in a sample that can be detected, but not necessarily quantified. The limit of detection is frequently confused with the sensitivity of the method. The sensitivity of an analytical method is the capability of the method to discriminate small differences in concentration or mass of the test analyte. In practical terms, sensitivity is the slope of the calibration curve that is obtained by plotting the response against the analyte concentration or mass. In chromatography, the detection limit is the injected amount that results in a peak with a height at least two or three times as high as the baseline noise level. Furthermore this signal/noise method describes three more methods (ICH):

- Visual inspection: The detection limit is determined by the analysis of samples with known concentrations of analyte and by establishing the minimum level at which the analyte can be reliably detected.

- Standard deviation of the response based on the standard deviation of the blank: Measurement of the magnitude of analytical background response is performed by analyzing an appropriate number of blank samples and calculating the standard deviation of these responses.

- Standard deviation of the response based on the slope of the calibration curve: A specific calibration curve is studied using samples containing an analyte in the range of the limit of detection. The residual standard deviation of a regression line, or the standard deviation of y-intercepts of regression lines, may be used as the standard deviation.

\subsubsection{Limit of quantitation}

The limit of quantitation is the minimum injected amount that produces quantitative measurements in the target matrix with acceptable precision in chromatography, typically requiring peak heights 10 to 20 times higher than the baseline noise. If the required precision of the method at the limit of quantitation has been specified, the EURACHEM approach can be used. A number of samples with decreasing amounts of the analyte are injected six times. The calculated RSD percent of the precision is plotted against the analyte amount.

The amount that corresponds to the previously defined required precision is equal to the limit of quantitation. It is important to use not only pure standards for this test but also spiked matrices that closely represent the unknown samples. For the limit of quantitation, the $\mathrm{ICH}$ recommends, in addition to the procedures as described above, the visual inspection and the standard deviation of the response and the slope of the calibration curve. Any results of limits of detection and quantitation measurements must be verified by experimental tests with samples containing the analytes at levels across the two regions. It is equally important to assess other method validation parameters, such as precision, reproducibility and accuracy, close to the limits of detection and quantitation. Figure 2 illustrates both the limit of detection and the limit of quantitation.

\subsection{Precision}

Precision provides an indication of random errors and can be broken down into repeatability and intermediate precision. This procedure should only be performed when 


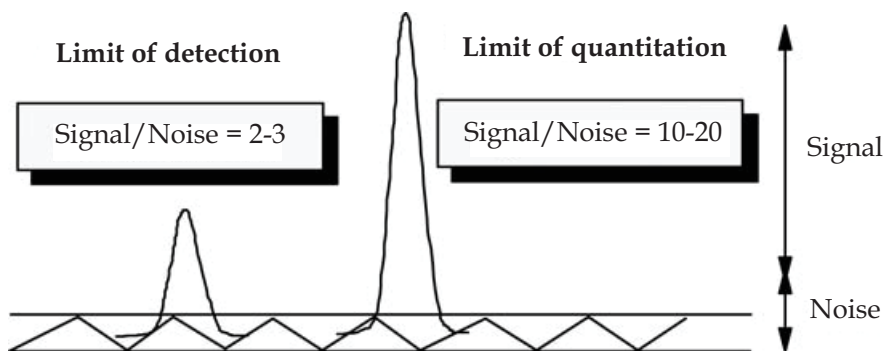

Fig. 2. Limit of detection and limit of quantitation via signal to noise

the entire analytical method procedure is finalized. Repeatability represents the simplest situation and involves analysis of replicates by the same analyst, generally one injection after the other. Repeatability tests are mandatory for all tests delivering numerical data. Repeatability is divided into two parts: injection repeatability and analysis repeatability (multiple preparations). Validation of the precision of an HPLC method occurs at three stages. The first stage is injection precision (injection repeatability) based on multiple injections of a single preparation of a sample on a particular sample on a given day. The set of criteria is given for area (\% area normalization) methods (drug substance and drug product) based on \%RSD of peak area. The second stage is analysis repeatability where multiple preparations and multiple injections of a sample are analyzed by the same chemist on the same day. The third stage is intermediate precision and is usually performed by different analysts, on a different system, on a different day on the same drug substance or drug product batch to determine the variability of the analytical test. The intermediate precision test may give indications to potential issues that may arise during method transfer. Relative standard deviation or coefficient of variation (Srel or \%RSD) is used to assess whether the adequate precision has been obtained. If automation is utilized, then an intermediate precision test is required to compare results obtained through manual testing versus automated testing (if all solvent composition and analyte concentrations of all actives are identical in both methods).

\subsubsection{Repeatability}

In addition to the system precision, this short-term variability includes the contributions from the sample preparation, such as weighing, aliquoting, dilution, extraction, homogenisation, and etc. Therefore, it is essential to apply the whole analytical procedure (as described in the control test), rather merely to injecting the same sample solution six times. This is also the reason for using authentic samples because only then can the analytical procedure be performed exactly as in the routine application. There may be exceptions, but these should be demonstrated or cautiously justified. For example, analysing degradants near of the quantitation limit, where the variance contribution of the sample preparation can be neglected, injection precision and repeatability are identical. For some applications, where precision can be regarded as less critical, such as in early development or if the variability demands only a small part of specification range (less than approximately $10 \%$ ), or if the expected content of impurities is far away from the specification limit, artificially prepared (spiked) samples may be used, allowing several validation characteristics (linearity, precision and accuracy) to be addressed simultaneously. Repeatability can be calculated using Eq.(1) and Eq. (2) from a larger number of repeatedly prepared samples (at least 6). 


$$
\begin{gathered}
s=\sqrt{\sum_{i=1}^{n}\left(x_{i}-\bar{x}\right)^{2} /(n-1)} \\
\% R S D=\frac{s}{\bar{x}} \cdot 100 \%
\end{gathered}
$$

Where $\mathrm{s}$ is the standard deviation, $\mathrm{xi}$ is the individual measurement in a set of $\mathrm{n}$ measurements and $\bar{x}$ is the mean of all the measurements. \%RSD is the standard deviation in percentage.

\subsubsection{Intermediate precision}

Intermediate precision includes the influence of additional random effects according to the intended use of the procedure in the same laboratory and can be regarded as an (initial) estimate for the long-term variability. Relevant factors, such as operator, instrument, and days should be varied. Intermediate precision is obtained from several independent series of applications of the (whole) analytical procedure to (preferably) authentic, identical samples. In some cases of relative techniques, the preparation and analysis of the reference standard is an important variability contribution. Therefore, it is not appropriate to determine intermediate precision from the peak area of the sample alone (analysed on different days or even several concentrations only), as it is sometimes reported in validation literature. Apart from ignoring the contribution of the reference standard, any signal shift of the instrument will be falsely interpreted as random variability. In order to reflect the expected routine variability properly, the calibration must be performed exactly as described in the control test. Reproducibility, according to the ICH definition, is obtained by varying further factors between laboratories and is also particularly important in the assessment of "official" compendia methods or whether the method is applied at different sites. However, understood in the long-term perspective, both intermediate precision and reproducibility approach each other, at least in the same company. Reproducibility from collaborative trials can be expected to include additional contributions due to a probably larger difference of knowledge, experience, equipment, and etc. among the participating laboratories.

\subsubsection{Reprodutibility}

Reproducibility represents the precision obtained between different laboratories. The objective is to verify that the method will provide the same results in different laboratories. The reproducibility of an analytical method is determined by analyzing aliquots from homogeneous lots in different laboratories with different analysts, and by using operational and environmental conditions that may differ from, but they are still within, the specified parameters of the method (interlaboratory tests). Validation of reproducibility is important whether the method is to be used in different laboratories. The main factors affecting the reprodutibility are:

- Differences in room temperature and humidity.

- Operators with different experience and thoroughness.

- Equipment with different characteristics, e.g., delay volume of an HPLC system.

- Variations in material and instrument conditions, e.g., HPLC, composition of mobile phase, $\mathrm{pH}$, and flow rate of mobile phase.

- Variation in experimental details not specified by the method.

- Equipment and consumables of different ages.

- Columns from different suppliers or different batches.

- Solvents, reagents, and other materials with varying quality. 


\subsection{Accuracy}

The accuracy of an analytical method is the extent to which test results generated by the method and the true value agree. Accuracy can also be described as the closeness of agreement between the value that is adopted, either as a conventional, true, or accepted reference value, and the value found. For the quantitative approaches, at least nine determinations across the specified range should be obtained, for example, three replicates at three concentration levels each. The percentage recovery or the difference between the mean and the accepted true value together with the confidence intervals are recommended.

The expected recovery (Table 7) depends on the sample matrix, the sample processing procedure, and the analyte concentration. The AOAC Guidelines for Single Laboratory Validation of Chemical Methods for Dietary Supplements and Botanicals (AOAC, 2002) includes a table with estimated recovery data as a function analyte concentration.

\begin{tabular}{lccc}
\hline Active Ingredient (\%) & Analyte ratio & Unit & Mean recovery $(\%)$ \\
\hline 100 & 1 & $100 \%$ & $98-102$ \\
$\geq 10$ & $10^{-1}$ & $10 \%$ & $98-102$ \\
$\geq 1$ & $10^{-2}$ & $1 \%$ & $97-103$ \\
$\geq 0.1$ & $10^{-3}$ & $0.1 \%$ & $95-105$ \\
$\geq 0.01$ & $10^{-4}$ & $100 \mathrm{ppm}$ & $90-107$ \\
$\geq 0.001$ & $10^{-5}$ & $10 \mathrm{ppm}$ & $80-110$ \\
$\geq 0.0001$ & $10^{-6}$ & $1 \mathrm{ppm}$ & $80-110$ \\
$\geq 0.00001$ & $10^{-7}$ & $100 \mathrm{ppb}$ & $80-110$ \\
$\geq 0.000001$ & $10^{-8}$ & $10 \mathrm{ppb}$ & $60-115$ \\
$\geq 0.0000001$ & $10^{-9}$ & $1 \mathrm{ppb}$ & $40-120$ \\
\hline
\end{tabular}

Table 7. Analyte recovery at different concentrations

In drug substance, it can be rather difficult to demonstrate accuracy for a drug substance appropriately, especially if no (independently) characterised reference standard is available. In addition, independent analytical procedures are often not readily found. Nevertheless, every effort should be made to identify a suitable method for comparison because this is the only way to verify accuracy objectively. Instead of quantitative comparison, the results could also be supported by another method, for example, the verification of a very high purity of a drug substance by differential scanning calorimetry. Sometimes in validation literature, recovery is reported for a drug substance. However, recovery from simple solutions does not provide meaningful information (at least if all determinations are traced back to a reference standard characterized with the same analytical procedure) and it, therefore, is not appropriate to demonstrate accuracy.

In drug product, the accuracy is usually validated by analysing a synthetic mixture of the drug product components which contain known amounts of a drug substance, also termed spiking or reconstituted drug product. The experimentally obtained amount of active substance is then compared to the true, added amount (recovery). It can be calculated either at each level separately as a percentage recovery or as a linear regression of the found analyte versus the added one (recovery function). Sometimes the term "recovery" is misused when reporting in active content of the drug product batches. This is misleading because in some cases, the true amount of active is influenced by manufacturing the variability and is not exactly known. Preferably, the result should be termed "\% label claim". The analyst should be aware of two important aspects regarding respect to recovery. First of all, it is based on the (validated) accuracy of the drug procedure substance otherwise 
the added amount will already be wrong. Secondly, by preparing the reconstituted drug product, the analyst deviates (more or less) from the routine analytical procedure. Of course, there is no other possibility of adding exactly known amounts, but consideration should be given to the possible implications. If, for example, solutions of the placebo are spiked with a stock solution of the active substance, the influence of the missed sample preparation steps, such as grinding, extracting, and etc. on the analysis should be considered. Here, the information obtained during the development of the method is very helpful (for example, homogeneity or extraction investigations). If some steps are of importance, any problems related to them will not influence the experimental recovery, and therefore, they are not identified.

\subsection{Robustness}

Robustness tests examine the effect that operational parameters have on the analysis results. For the determination of robustness, a number of method parameters are varied within a realistic range, and the quantitative influence of the variables is determined. If the influence of the parameter is within a previously specified tolerance, the parameter is said to be within the robustness of the method range. Obtaining data on these effects helps to assess whether a method needs to be revalidated, for instance, when one or more parameters are changed to compensate the column performance over time. In the $\mathrm{ICH}$ document, it is recommended to consider the evaluation of robustness of the method during the development phase, and any results that are critical for the method should be documented. In summary, an analyst must have a critical look at three different types of parameters when robustness and ruggedness are investigated:

- Internal parameters (e.g., temperature, $\mathrm{pH}$, and etc., in the case of HPLC).

- External parameters (e.g., different analysts, instruments, laboratories, and etc.).

- Basic parameters (e.g., stability of test solutions and etc.).

From these lists, one point becomes very clear. A test for robustness is an individual test and depends very much on the analytical technique and equipment applied. As a rule of thumb, it is recommended to examine at least those parameters which are part of the operational qualification of the respective equipment. Then, the set of parameters investigated in a robustness study can be arbitrarily extended to those specific to the method defined in the procedure. The usual way to performing robustness testing is first to define the parameters with reasonable maximum variation. Then, each parameter is successively varied whereas the others are held constant (at nominal setting). For instance, six parameters each one at two levels would require twelve experiments when one parameter is changed and the others are always set to nominal levels. If more parameters are included, more experiments must be conducted. This classical approach is called one-factor-a-time (OFAT) approach. Certainly, this kind of robustness testing has disadvantages, such as many experiments and time. In addition, only limited information is made available from such studies, since possible interactive effects which occur when more than one parameter (factor) is varied, cannot be identified. Nowadays, an experimental design approach (DOE: design of experiment) is often preferred for robustness testing. The aim of an experimental design is to obtain as much as possible relevant information in the shortest time from a limited number of experiments. Different designs can be used in robustness testing, e.g. including full- and fractional - factorial designs as well as Plackett-Burman designs. The latter have become very popular in method robustness testing during recent years. The choice of a design depends on the purpose of the test and the number of involved factors. Experimental designs in robustness testing can be employed for all analytical techniques. 


\section{Conclusion}

This chapter described the fundamentals and figures of merit for method validation in pharmaceutical analysis. The validation process is to confirm that the method is suited for its intended purpose and to prove the capabilities of the test method. The definitions of method validation parameters are well explained by health authorities. Although the requirements of validation have been clearly documented by regulatory authorities, the approach to validation is varied and opened to interpretation, and validation requirements differ during the development process of pharmaceuticals.

\section{Acknowledgment}

The authors acknowledge Instituto de Aperfeiçoamento Farmacêutico (IAF) for the scientific discussions and financial support.

\section{References}

AOAC International. (2002). AOAC Guidelines for Single Laboratory Validation of Chemical Methods for Dietary Supplements and Botanicals, Arlington, VA. Available from http://www.aoac.org/Official_Methods/slv_guidelines.pdf

BRASIL. (2003). Resolução RE n.899, de 29 de maio de 2003. Determina a publicação do Guia para validação de métodos analíticos e bioanalíticos. Diário Oficial da União, Brasília, 02 de junho de 2003. Available from http://www.anvisa.gov.br/legis/resol/2003/re/899_03re.htm

CDER Guideline on Validation of Chromatographic Methods. (1994). Reviewer Guidance of Chromatographic Methods, US Food and Drug Administration, Centre for Drugs and Biologics, Department of Health and Human Services

EURACHEM. (1998). A Laboratory Guide to Method Validation and Related Topics: The Fitness for Purpose of Analytical Methods, ISBN 0-948926-12-0, Teddington, Middlesex, United Kigdom.

Guidelines for Submitting Samples and Analytical Data for Methods Validation. (1987). US Food and Drug Administration, Centre for Drugs and Biologics, Department of Health and Human Services.

International Conference on the Harmonization of Technical Requirements for Registration of Pharmaceuticals for Human Use (ICH). Validation of Analytical Procedures: Text and Methodology Q2 (R1). (2005). Available from

http://www.ich.org/fileadmin/Public_Web_Site/ICH_Products/Guidelines/Qua lity/Q2_R1/Step4/Q2_R1_Guideline.pdf

Klick, S.; Muijselaar, P.G.; Waterval, J.; Eichinger, T.; Korn, C.; Gerding, T.K.; Debets, A.J.; Sänger-van de Griend; van den Beld, C.; Somsen, G.W and De Jong, G.J. (2005). Toward a Generic Approach for Stress Testing of Drug Substances and Drug Product. Pharmaceutical Technology, Vol.29, No.2, pp. 48-66, ISSN 1543-2521

United States Pharmacopeia. (2011). Chapter 1225: Validation of Compendial Methods. United States Pharmacopeia 33, National Formulary 28. Rockville, MD. 


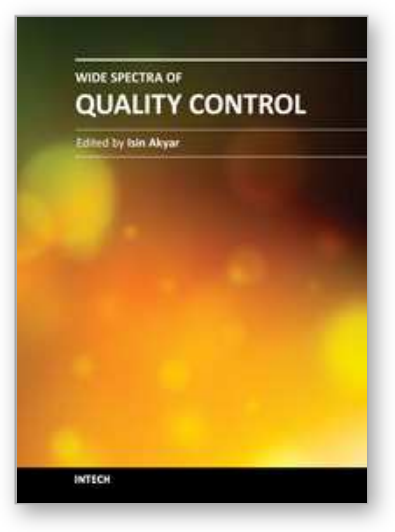

\author{
Wide Spectra of Quality Control \\ Edited by Dr. Isin Akyar
}

ISBN 978-953-307-683-6

Hard cover, 532 pages

Publisher InTech

Published online 07, July, 2011

Published in print edition July, 2011

Quality control is a standard which certainly has become a style of living. With the improvement of technology every day, we meet new and complicated devices and methods in different fields. Quality control explains the directed use of testing to measure the achievement of a specific standard. It is the process, procedures and authority used to accept or reject all components, drug product containers, closures, in-process materials, packaging material, labeling and drug products, and the authority to review production records to assure that no errors have occurred. The quality which is supposed to be achieved is not a concept which can be controlled by easy, numerical or other means, but it is the control over the intrinsic quality of a test facility and its studies. The aim of this book is to share useful and practical knowledge about quality control in several fields with the people who want to improve their knowledge.

\title{
How to reference
}

In order to correctly reference this scholarly work, feel free to copy and paste the following:

Pedro Lopez Garcia, Ernesto Buffoni, Fabio Pereira Gomes and Jose Luis Vilchez Quero (2011). Analytical Method Validation, Wide Spectra of Quality Control, Dr. Isin Akyar (Ed.), ISBN: 978-953-307-683-6, InTech, Available from: http://www.intechopen.com/books/wide-spectra-of-quality-control/analytical-method-validation

\section{INTECH}

open science | open minds

\section{InTech Europe}

University Campus STeP Ri

Slavka Krautzeka 83/A

51000 Rijeka, Croatia

Phone: +385 (51) 770447

Fax: +385 (51) 686166

www.intechopen.com

\section{InTech China}

Unit 405, Office Block, Hotel Equatorial Shanghai

No.65, Yan An Road (West), Shanghai, 200040, China 中国上海市延安西路65号上海国际贵都大饭店办公楼 405 单元

Phone: +86-21-62489820

Fax: $+86-21-62489821$ 
(C) 2011 The Author(s). Licensee IntechOpen. This is an open access article distributed under the terms of the Creative Commons Attribution 3.0 License, which permits unrestricted use, distribution, and reproduction in any medium, provided the original work is properly cited. 